\title{
Contextual Fear Memories Formed in the Absence of the Dorsal Hippocampus Decay Across Time
}

\author{
Moriel Zelikowsky, ${ }^{1,2,3}$ Stephanie Bissiere, ${ }^{1,2,3,4}$ and Michael S. Fanselow ${ }^{1,2,3}$ \\ Departments of ${ }^{1}$ Psychology, ${ }^{2}$ Psychiatry and Biobehavioral Sciences, and ${ }^{3}$ The Brain Research Institute, University of California, Los Angeles, Los Angeles, \\ California 90095, and ${ }^{4}$ Neuropeptides and Behavioural Neuroscience Divisions, Florey Neuroscience Institutes, University of Melbourne, Melbourne, \\ Victoria 3010, Australia
}

\begin{abstract}
Mammals suffering damage to the hippocampus display a dramatic loss of explicit, recently formed memories (retrograde amnesia). In contrast, deficits in the ability to form new memories following hippocampal damage (anterograde amnesia) can be overcome with sufficient training. By combining contextual fear conditioning with lesions of the dorsal hippocampus in rats, we discovered that while animals can form long-term contextual fear memories in the absence of the hippocampus, these memories decay with time, lacking the permanence that is a hallmark characteristic of normal fear memories. These findings indicate that while it is initially possible to acquire explicit memories when the hippocampus is compromised, these memories cannot transfer from a recent to remote state. This suggests that memories formed outside the hippocampus may nevertheless require the hippocampus to undergo systems consolidation, which has important clinical implications for the treatment of memory disorders.
\end{abstract}

\section{Introduction}

The hippocampus is a critical structure for many forms of memory. Loss of the hippocampus due to pathology or surgery in humans or from targeted experimental ablations in animals produces a profound amnesia (Squire, 1992). Memories for events that occurred prior to damage are lost in a manner that is temporally graded (retrograde amnesia), such that memory loss is greatest for events proximal to the insult and lessens for older memories (Zola-Morgan and Squire, 1990; Kim and Fanselow, 1992; Anagnostaras et al., 1999). Impairments in the ability to generate new memories (anterograde amnesia) also occur following hippocampal damage (Clark et al., 2002) or disruption of synaptic plasticity within the hippocampus at the time of learning (Morris et al., 1986; Young et al., 1994).

This set of findings suggests that the hippocampus is not only critical for the initial formation and storage of memories, but also participates in their ability to be gradually stabilized in other brain regions, a process referred to as systems consolidation, which can take weeks, months, or even years (Squire, 2004). Importantly, systems consolidation is distinct from cellular consolidation (Debiec et al., 2002), which provides a mechanism for the transition from active short-term memory to passive long-term memory in a process that occurs for several hours after the acquisition or retrieval of information (Schafe et al., 1999; Nader et al., 2000).

Received Aug. 24, 2011; revised Dec. 15, 2011; accepted Jan. 18, 2012.

Author contributions: M.Z. and M.S.F. designed research;M.Z. performed research; M.Z. contributed unpublished reagents/analytic tools; M.Z. analyzed data; M.Z., S.B., and M.S.F. wrote the paper.

This research was supported by National Institute of Mental Health Grant R01 MH062122 (to M.S.F.) and a Charles F. Scott Endowed Fellowship (to M.Z.). We thank Timothy Hast and Rebecca Bennett for technical support.

Correspondence should be addressed to Michael S. Fanselow, Department of Psychology, University of California, Los Angeles, 405 Hilgard Avenue, Los Angeles, CA 90095-1563. E-mail: fanselow@psych.ucla.edu.

DOI:10.1523/JNEUROSCI.4339-11.2012

Copyright $\odot 2012$ the authors $\quad 0270-6474 / 12 / 323393-05 \$ 15.00 / 0$
Initially it was thought that the magnitude of anterograde amnesia and retrograde amnesia was proportional (Squire and Alvarez, 1995). However, studies using contextual fear conditioning in rodents have shown that while retrograde amnesia is invariably pronounced, anterograde amnesia can be readily overcome with modest increases in training parameters (Maren et al., 1997; Frankland et al., 1998; Wiltgen et al., 2006). This has led to the view that while the hippocampus is normally used for contextual fear conditioning, in the absence of the hippocampus other regions may compensate, albeit less efficiently (Fanselow, 2010).

Although contextual fear memories can be formed in the absence of the hippocampus, it is not clear whether memories formed using alternate circuitry maintain the same properties as those formed with the hippocampus intact. One classic hallmark of normal fear memories is that they last the lifespan of the rat without diminution (Gale et al., 2004). However, the durability of contextual fear memories acquired in the absence of the hippocampus remains unknown. Ascertaining whether such memories retain permanency would provide insight into the brain mechanisms underlying compensation, the necessity of the hippocampus in systems consolidation, and the optimization of targeted clinical approaches for the treatment of memory disorders.

Therefore, we tested whether contextual fear memories formed following damage to the dorsal hippocampus (DH) were as stable as those formed with the hippocampus intact. We discovered that rats conditioned in the absence of the dorsal hippocampus were able to acquire contextual fear and retain this memory across a $1 \mathrm{~d}$ retention interval; however, as this interval was extended, contextual fear expression began to fade. Thus, memories formed using compensatory structures may retain the ability to undergo cellular consolidation but lack the ability to undergo the consolidation process necessary to allow expression of these memories at remote time points. 


\section{Materials and Methods}

Subjects. The subjects were 60 naive, adult male Long-Evans rats, initially weighing 270-300 g, purchased from Harlan. Rats were individually housed and maintained on a $12 \mathrm{~h}$ light/dark cycle with access to food and water ad libitum (all behavioral testing conducted during the light portion of the cycle). Animals were handled daily (1-2 min per rat) for at least 1 week before the start of surgery and behavioral training. The procedures used in this experiment were in accordance with policy set and approved by the Institutional Animal Care and Use Committee of the University of California, Los Angeles.

Surgery. Rats were anesthetized with sodium pentobarbital $(65 \mathrm{mg} / \mathrm{kg}$, i.p.) and medicated with atropine sulfate $(0.4 \mathrm{mg} / \mathrm{kg}$, i.p.). Each rat was shaved across the head, and their eyes were coated with a hydrating ointment. Animals were mounted into stereotaxic instruments (Kopf Instruments), and the scalp was cleaned (70\% ethyl alcohol and Betadine), incised, and retracted. The skull was adjusted so that bregma and lambda were in the same horizontal plane. Four small holes (two per side) were drilled into the skull to allow for a stainless steel injector cannula (33 gauge) aimed at the dorsal hippocampus to be positioned 2.8 $\mathrm{mm}$ posterior, $1.6 \mathrm{~mm}$ lateral, $3.5 \mathrm{~mm}$ ventral to bregma (rostral coordinates), and $4.2 \mathrm{~mm}$ posterior, 2.6 lateral, and $3.5 \mathrm{~mm}$ ventral to bregma (caudal coordinates). Injection cannulae (33 gauge) were attached to a 5 $\mu \mathrm{l}$ microsyringe (Hamilton Instruments) via polyethylene tubing (PE20) and inserted into guide cannulae (28 gauge) attached to the arms of the stereotax. Microsyringes were mounted into a syringe pump (Harvard Apparatus) for controlled microinfusions of NMDA (20 mg/ml; SigmaAldrich), dissolved in $0.01 \mathrm{M}$ PBS. Cannulae were lowered, and infusions of $0.4 \mu \mathrm{l}$ of NMDA/site were made across $4 \mathrm{~min}(0.1 \mu \mathrm{l} / \mathrm{min}$ rate). Cannulae remained in place for an additional $2 \mathrm{~min}$ to allow for adequate NMDA diffusion and reduction of backflow. Sham surgeries were identical except that injection cannulae were not lowered, and infusions were not made. Following infusions, incisions were closed with stainless steel wound clips, and animals were given intraperitoneal injections of the analgesic/anti-inflammatory ketoprofen $(2 \mathrm{mg} / \mathrm{kg})$ and placed on heating pads until they recovered from anesthesia. Ketoprofen injections were continued for an additional $2 \mathrm{~d}$ postsurgery. In addition, rats were given the antibiotic trimethoprim sulfa in their drinking water, weighed, monitored, and handled for 1 week following surgery. Rats were allowed a total of 11-13 d of recovery before behavioral training.

Apparatus. All behavioral training was performed in the same "context," which was composed of a set of four identical fear conditioning chambers $(30 \times 25 \times 25 \mathrm{~cm}$, Med Associates $)$, equipped with a Med Associates VideoFreeze system. Individual boxes were enclosed in sound-attenuating chambers (Med Associates), and the set was contained in an individual, dedicated experimental room, which provided a unique spatial location. Each box had aluminum sidewalls and a Plexiglas rear wall with blue dots. The grid floor consisted of 16 stainless steel rods (4.8 mm thick) spaced $1.6 \mathrm{~cm}$ apart (center to center; Contextual Conditioning System, Med Associates). Pans underlying each box were sprayed with a thin film of Simple Green to provide the context with a scent. Chambers were individually lit from above with white house lights and cleaned with $70 \%$ isopropyl alcohol in between squads. Individual fans mounted above each chamber were turned on to provide background noise $(60 \mathrm{~dB})$. The experimental room in which chambers were located was brightly lit with overhead lights. Animals were transported to chambers in squads of four in their homecages, which were slid onto hanging racks mounted to a portable cart and covered with a white sheet. Chambers were cleaned with a $10 \%$ bleach solution following each day of behavioral testing.

Fear conditioning. Ten to $14 \mathrm{~d}$ following surgery (recovery period), rats were transported to the conditioning context in squads of four and placed into individual experimental chambers. Following a $180 \mathrm{~s}$ baseline period of exposure to the context, rats were fear conditioned using four, $0.9 \mathrm{~mA}, 2 \mathrm{~s}$ footshocks. Footshocks were separated by an $88 \mathrm{~s}$ intertrial interval. Following the final shock, rats were given 60 additional seconds in the context before being transported back to the vivarium. Rats were then brought back to the context for an $8 \mathrm{~min}$ exposure session to test for contextual fear memory either $1,3,10$, or $30 \mathrm{~d}$ later (see Fig. $2 A$ ). This
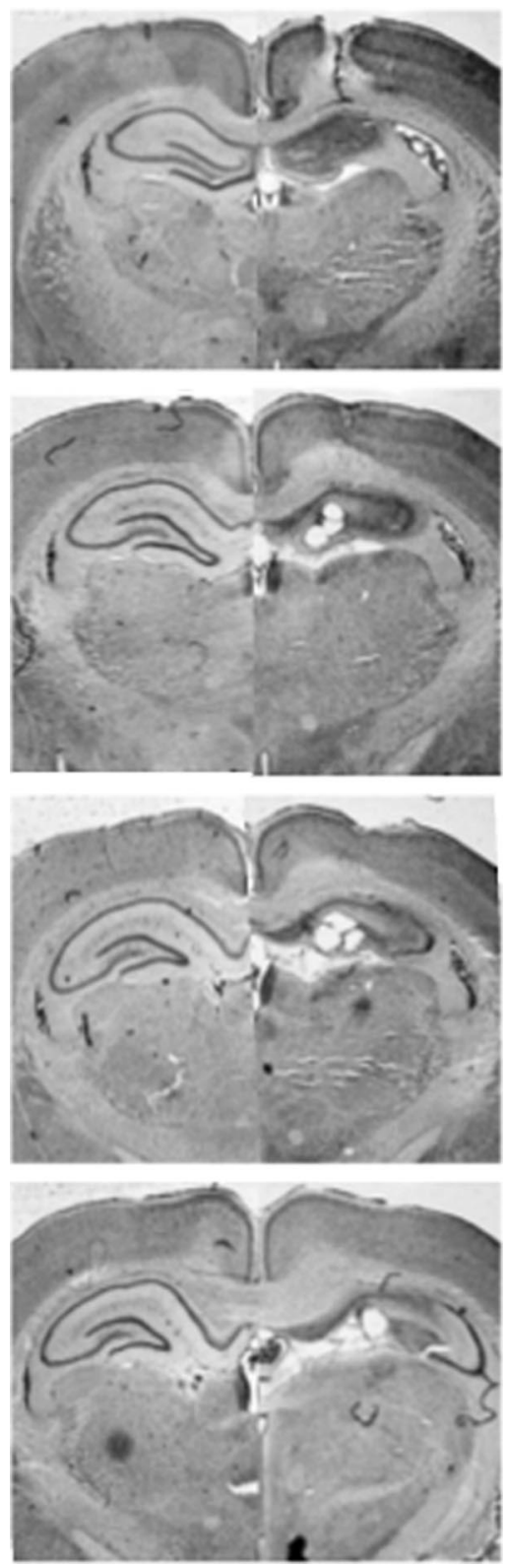

Figure 1. Representative photomicrographs show cresyl violet-stained coronal brain sections following excitotoxic lesions of the dorsal hippocampus (right) compared with sham controls (left). From top to bottom, the sections are 1.60, 2.60, 3.60, and $4.60 \mathrm{~mm}$ posterior to bregma.

resulted in the following eight experimental groups of a 2 (surgery) $\times 4$ (retention interval) factorial design: Sham-1, Sham-3, Sham-10, Sham30, DH-1, DH-3, DH-10, DH-30.

Histology. Following behavioral testing, animals were anesthetized and transcardially perfused with PBS followed by $4 \%$ paraformaldehyde (PFA). Brains were extracted and placed in 4\% PFA overnight. The following day they were cryoprotected in a $30 \%$ sucrose solution for $72 \mathrm{~h}$. The brains were then frozen $\left(-20^{\circ} \mathrm{C}\right)$ and sectioned on a cryostat $(50$ $\mu \mathrm{m})$. Every third section $(150 \mu \mathrm{m})$ was collected and dry mounted on a microscope slide. Sections were then stained for nissl bodies (cresyl violet), lesions were verified, and images were captured using bright-field microscopy.

Data analysis. Rats freeze in fear-provoking contexts, and we relied on this freezing behavior to assess fear memory. To do this we used an automated near-infrared video tracking equipment and computer software (VideoFreeze, Med Associates). Video was recorded at $30 \mathrm{frames} / \mathrm{s}$, and the software calculated the frame-to-frame change in grayscale val- 
A

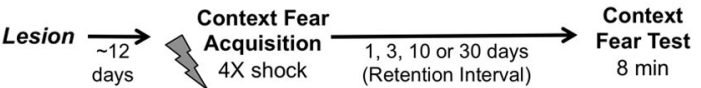

B

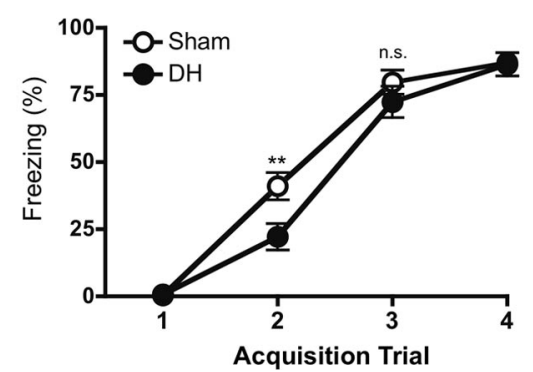

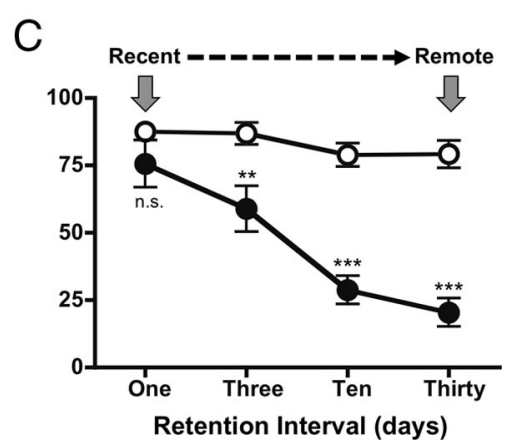

Figure 2. Longevity of a contextual fear memory formed in the absence of the hippocampus. $\boldsymbol{A}$, Experimental design. $\boldsymbol{B}$, Contextual fear acquisition. Mean ( \pm SEM) freezing to the context during the 30 s period preceding each footshock (trial) for rats with an intact (sham) or damaged dorsal hippocampus (DH). All animals acquired contextual fear, regardless of lesion condition. $\boldsymbol{C}$, Context fear test. Mean ( \pm SEM) freezing to the context averaged over the 8 min context exposure test are displayed. Rats were tested 1, 3, 10, or $30 \mathrm{~d}$ following acquisition (retention interval). The $1 \mathrm{~d}$ test represents our measure of "recent" long-term memory, the $30 \mathrm{~d}$ test represents our measure of "remote" long-term memory. Sham rats maintained similar levels of freezing independent of retention interval. Animals conditioned in the absence of the DH exhibited compensatory contextual fear expression $1 \mathrm{~d}$ following training; however, the expression of this memory decayed dramatically across time. (n.s., not significant, $p>$ $0.05 ;{ }^{* *} p<0.01 ;{ }^{* * *} p<0.001$ ).

ues for each pixel and summed these values for a frame. This value was compared with change values calculated when no animal was present (noise) to obtain an "activity score" for each frame. Based on previous validation with hand scoring by trained human observers that defined freezing as the absence of all movement except that necessitated by respiration (correlation of $r>0.9$ between automated system and highly trained human observers), an instance of freezing was defined as an activity score $<50$ for longer than $1 \mathrm{~s}$. For fear acquisition, average freezing across the $30 \mathrm{~s}$ before each footshock administration was scored and analyzed. For fear memory at test, freezing across the entire $8 \mathrm{~min}$ of context exposure was scored and analyzed. Freezing data were statistically analyzed using between-subjects ANOVAs and repeated-measures (trial) ANOVAs where appropriate. Post hoc tests (Bonferroni) were performed following significant findings. The level of significance used for all analyses was $p<0.05$.

\section{Results}

To investigate the durability of memories formed in the absence of the dorsal hippocampus, rats were given excitotoxic lesions of the dorsal hippocampus or sham surgery (controls) before context fear conditioning. We focused specifically on the dorsal as opposed to ventral region of the hippocampus because of the dorsal hippocampus' role in memory generally and in contextual fear in particular (Kim and Fanselow, 1992; Fanselow and Dong, 2010). Moreover, lesions of the dorsal hippocampus produce the same pattern of results for contextual fear memory as those produced by lesions of the entire hippocampal complex (Wiltgen et al., 2006). We compromised the DH using permanent lesions as opposed to temporary inactivations because compensatory contextual fear memories have been demonstrated following the former (Maren et al., 1997; Wiltgen et al., 2006) but not the latter (Sanders and Fanselow, 2003; Matus-Amat et al., 2004). Moreover, inactivation of the $\mathrm{DH}$ has been shown to work well when one is targeting fear acquisition but does not affect consolidation (Kim et al., 1991).

Representative photomicrographs of a DH-lesioned rat compared with sham control are displayed in Figure 1. The extent of NMDA-induced excitotoxic lesions of the $\mathrm{DH}$ was consistent with those previously reported in our laboratory (Quinn et al., 2008). Rats with insufficient bilateral damage restricted to the
DH were not included. Based on this criterion, two animals were excluded. This resulted in the following group sizes: Sham-1, $n=8$; Sham-3, $n=6$; Sham-10, $n=8$; Sham-30, $n=8$; DH- $1, n=7$; DH-3, $n=6$; DH-10, $n=8$; DH-30, $n=7$.

Following pretraining lesions of the $\mathrm{DH}$ or sham surgery, animals were allowed to recover for 11-13 d before undergoing behavioral testing. All rats underwent contextual fear acquisition and testing (see Fig. $2 \mathrm{~A}$ for design). Figure $2 B$ displays freezing across the acquisition session in sham and $\mathrm{DH}$ lesioned rats. A repeated-measures (trial) $\times$ lesion ANOVA revealed that both the sham and lesion groups were able to acquire contextual fear across conditioning trials $\left(F_{(3,168)}=276.7, p<0.0001\right)$ with an interaction between trial and lesion condition $\left(F_{(3,168)}=3.34, p<0.05\right)$.

Consistent with previous findings, rats showed a deficit in contextual fear expression following a single trial of conditioning (trial 2: $t=3.23 ; p<0.01$ ). However, this deficit disappeared with additional trials, consistent with the idea that contextual fear can be acquired in the absence of the hippocampus but less efficiently (Wiltgen et al., 2006). There was no effect of lesion before any shock administration (trial $1: t=0.04 ; p>0.05$ ), demonstrating that hippocampal lesions themselves did not affect baseline freezing.

Animals were tested for contextual fear memory 1, 3, 10, or $30 \mathrm{~d}$ following fear acquisition (Fig. $2 C$ ). We chose $1 \mathrm{~d}$ as the moderate retention interval as it is well outside the period of cellular consolidation and is commonly thought to be an interval appropriate for testing "recent" long-term memory (Schafe et al., 1999). To test for a possible failure in the retention of these memories, we examined contextual fear following a $30 \mathrm{~d}$ retention interval, as retrograde amnesia studies suggest that this is a period over which systems consolidation occurs (Kim and Fanselow, 1992). Additional $3 \mathrm{~d}$ and $10 \mathrm{~d}$ retention intervals were used to examine whether changes in fear expression across time were graded.

A two-way (lesion $\times$ retention interval) ANOVA revealed an overall significant effect of lesion, retention interval, and an interaction of these factors (lesion: $F_{(1,50)}=86.29, p<0.0001$; interval: $F_{(3,50)}=14.90, p<0.0001$; interaction: $F_{(3,50)}=7.31$, $p<0.001)$. Contextual fear expression following a $1 \mathrm{~d}$ retention interval was not different between sham and DH-lesioned animals, demonstrating that both groups were able to form a longterm contextual fear memory $(t=1.52 ; p>0.05)$. These findings are consistent with those previously reported (Wiltgen et al., 2006).

However, when this retention interval was extended, the ability for DH-lesioned animals to maintain a level of fear that was comparable to controls was dramatically reduced ( $3 \mathrm{~d}: t=3.20$, $p<0.01 ; 10 \mathrm{~d}: t=6.57, p<0.001 ; 30 \mathrm{~d}: t=7.45, p<0.001)$. Moreover, there was a significant simple main effect of retention interval for rats with $\mathrm{DH}$ lesions but not for sham controls $(\mathrm{DH}$ : $F_{(3,27)}=13.98, p<0.0001$; sham: $\left.F_{(3,28)}=1.42, p>0.05\right)$, indicating that the loss of contextual fear expression across time was specific to DH-lesioned animals. These results indicate that expression of contextual fear memories formed in the absence of the hippocampus is attenuated with the passage of time. 


\section{Discussion}

In this study, we found that rodents that acquire contextual fear memories in the absence of the dorsal hippocampus fail to express these memories as they become remote. These data suggest that contextual fear memories formed in the absence of the dorsal hippocampus may lack the persistence of those formed with the hippocampus intact. In line with previous findings, they show that animals can overcome the amnestic effects of pretraining $\mathrm{DH}$ lesions provided adequate training is given (Wiltgen et al., 2006). However, our results are the first to reveal that the ability to overcome anterograde amnesia may be dependent on the length of time between training and test, such that recently acquired contextual fear memories remain intact while the expression of such memories at remote time points is dramatically impaired. Thus, explicit memories formed using alternate, compensatory structures seem to lack the permanence characteristic of normal contextual fear memories (Gale et al., 2004). One way to interpret these findings is that contextual fear memories formed in the absence of the $\mathrm{DH}$ are unable to be consolidated in the same way as those formed with the $\mathrm{DH}$ intact.

These findings suggest that even when contextual fear memories rely on regions outside the $\mathrm{DH}$ for initial memory formation, the hippocampus is vital for the transformation of these memories from a recent to remote state. That is, the DH may be required for the long-term consolidation of explicit memories, regardless of the structure in which these memories were initially established. Thus, the properties required of an explicit memory in order for it to be subject to cellular consolidation may not necessarily be sufficient for that memory to be permanently consolidated.

We demonstrate that in order for a contextual fear memory to become permanent, the $\mathrm{DH}$ must be intact. This implies that the compensatory structures recruited in the absence of the $\mathrm{DH}$ are incapable of permanently holding onto these memories, suggesting that they are more fragile in nature. The involvement of the $\mathrm{DH}$ in the ability for long-term memories to be stored permanently is supported by studies showing that memories formed in the remote past can be disrupted by blocking CA3 output, therein disturbing the integrity of the tri-synaptic pathway and the ripple-associated reactivation of hippocampal memory (Nakashiba et al., 2009). Additionally, it has been shown that the prefrontal cortex is phase-locked to hippocampal theta (Siapas et al., 2005), and that ablation of NMDARs on interneurons (Korotkova et al., 2010) or blockade of electrical communication between interneurons in the hippocampus (Bissiere et al., 2011) disrupts hippocampal synchrony and hippocampus-dependent memory. These findings support the idea that an intact hippocampus is essential for the permanent consolidation of a memory or, at the least, that memories formed using alternate circuitry are not formed in neuronal ensembles that have access to the appropriate synchrony, electrical communication, or intracellular signaling properties required for stable storage.

Surprisingly, the straightforward prediction that memories formed in the absence of the hippocampus lack permanence has never been tested. Two factors likely contribute to the difficulty in conducting such a test. One is that as the retention interval between training and testing increases, normal forgetting in the intact controls can obscure a failure of systems consolidation with hippocampal damage (Zola-Morgan and Squire, 1990). A second issue is that tasks that take several days to acquire do not allow a clear separation between the period of acquisition and systems consolidation. Thus, an ideal task to test for the longevity of memories is one that can be acquired in a single brief session and is stable across time. By using contextual fear conditioning, we were able to employ a task that satisfied both of these requirements.

Finally, our findings dovetail nicely with those previously reported examining contextual fear in the absence of the basolateral amygdala (BLA). That is, the BLA is integral to fear learning and memory (Fanselow and LeDoux, 1999), and damage to the BLA results in a massive deficit in fear acquisition and expression (Maren et al., 1996). However, animals are able to learn and express context fear following BLA damage, provided they are trained using a very robust acquisition protocol (Maren, 1999; Ponnusamy et al., 2007; Poulos et al., 2010). In addition, contextual fear memories formed in the absence of the BLA do not persist across time (Poulos et al., 2009). Thus, our results for the $\mathrm{DH}$ parallel those found for the BLA, suggesting that both the $\mathrm{DH}$ and BLA are essential components of the circuitry required for a contextual fear memory to become permanent.

These findings imply a general conclusion about compensation following brain damage: namely, that learning and memory can occur in the absence of the relevant primary structure(s), but that learning is less efficient, and that the memories formed decay with time. Therefore, when the primary circuit for a specific task is compromised, not only does it seem to be a general property of the brain that an alternate circuit be recruited to compensate (Fanselow, 2010), but it may also be a general finding that these compensatory memories fade with time. While further experiments examining whether the same pattern is maintained across different hippocampus-dependent tasks and preparations are warranted, the question is most relevant in situations where expression of memory remains unabated with time.

These results also have important clinical implications. They suggest that memories normally thought to involve the hippocampus may be formed following hippocampal damage, but that these memories are more fragile, failing to persist across time. Thus, clinical approaches toward reducing or overcoming amnesia could benefit from developing methods to promote the longevity of memories formed following brain damage. Such advances would be facilitated by the identification of the brain regions underlying contextual memories formed in the absence of the hippocampus.

\section{References}

Anagnostaras SG, Maren S, Fanselow MS (1999) Temporally graded retrograde amnesia of contextual fear after hippocampal damage in rats: within-subjects examination. J Neurosci 19:1106-1114.

Bissiere S, Zelikowsky M, Ponnusamy R, Jacobs NS, Blair HT, Fanselow MS (2011) Electrical synapses control hippocampal contributions to fear learning and memory. Science 331:87-91.

Clark RE, Broadbent NJ, Zola SM, Squire LR (2002) Anterograde amnesia and temporally graded retrograde amnesia for a nonspatial memory task after lesions of hippocampus and subiculum. J Neurosci 22:4663-4669.

Debiec J, LeDoux JE, Nader K (2002) Cellular and systems reconsolidation in the hippocampus. Neuron 36:527-538.

Fanselow MS (2010) From contextual fear to a dynamic view of memory systems. Trends Cogn Sci 14:7-15.

Fanselow MS, Dong HW (2010) Are the dorsal and ventral hippocampus functionally distinct structures? Neuron 65:7-19.

Fanselow MS, LeDoux JE (1999) Why we think plasticity underlying Pavlovian fear conditioning occurs in the basolateral amygdala. Neuron 23:229-232.

Frankland PW, Cestari V, Filipkowski RK, McDonald RJ, Silva AJ (1998) The dorsal hippocampus is essential for context discrimination but not for contextual conditioning. Behav Neurosci 112:863-874.

Gale GD, Anagnostaras SG, Godsil BP, Mitchell S, Nozawa T, Sage JR, Wiltgen B, Fanselow MS (2004) Role of the basolateral amygdala in the stor- 
age of fear memories across the adult lifetime of rats. J Neurosci 24:3810-3815.

Kim JJ, Fanselow MS (1992) Modality-specific retrograde amnesia of fear. Science 256:675-677.

Kim JJ, DeCola JP, Landeira-Fernandez J, Fanselow MS (1991) N-methylD-aspartate receptor antagonist APV blocks acquisition but not expression of fear conditioning. Behav Neurosci 105:126-133.

Korotkova T, Fuchs EC, Ponomarenko A, von Engelhardt J, Monyer H (2010) NMDA receptor ablation on parvalbumin-positive interneurons impairs hippocampal synchrony, spatial representations, and working memory. Neuron 68:557-569.

Maren S (1999) Neurotoxic basolateral amygdala lesions impair learning and memory but not the performance of conditional fear in rats. J Neurosci 19:8696-8703.

Maren S, Aharonov G, Fanselow MS (1996) Retrograde abolition of conditional fear after excitotoxic lesions in the basolateral amygdala of rats: absence of a temporal gradient. Behav Neurosci 110:718-726.

Maren S, Aharonov G, Fanselow MS (1997) Neurotoxic lesions of the dorsal hippocampus and Pavlovian fear conditioning in rats. Behav Brain Res 88:261-274.

Matus-Amat P, Higgins EA, Barrientos RM, Rudy JW (2004) The role of the dorsal hippocampus in the acquisition and retrieval of context memory representations. J Neurosci 24:2431-2439.

Morris RG, Anderson E, Lynch GS, Baudry M (1986) Selective impairment of learning and blockade of long-term potentiation by an N-methyl-Daspartate receptor antagonist, AP5. Nature 319:774-776.

Nader K, Schafe GE, LeDoux JE (2000) The labile nature of consolidation theory. Nat Rev Neurosci 1:216-219.

Nakashiba T, Buhl DL, McHugh TJ, Tonegawa S (2009) Hippocampal CA3 output is crucial for ripple-associated reactivation and consolidation of memory. Neuron 62:781-787.

Ponnusamy R, Poulos AM, Fanselow MS (2007) Amygdala-dependent and amygdala-independent pathways for contextual fear conditioning. Neuroscience 147:919-927.
Poulos AM, Li V, Sterlace SS, Tokushige F, Ponnusamy R, Fanselow MS (2009) Persistence of fear memory across time requires the basolateral amygdala complex. Proc Natl Acad Sci U S A 106:11737-11741.

Poulos AM, Ponnusamy R, Dong HW, Fanselow MS (2010) Compensation in the neural circuitry of fear conditioning awakens learning circuits in the bed nuclei of the stria terminalis. Proc Natl Acad Sci U S A 107: 14881-14886.

Quinn JJ, Ma QD, Tinsley MR, Koch C, Fanselow MS (2008) Inverse temporal contributions of the dorsal hippocampus and medial prefrontal cortex to the expression of long-term fear memories. Learn Mem 15:368-372.

Sanders MJ, Fanselow MS (2003) Pre-training prevents context fear conditioning deficits produced by hippocampal NMDA receptor blockade. Neurobiol Learn Mem 80:123-129.

Schafe GE, Nadel NV, Sullivan GM, Harris A, LeDoux JE (1999) Memory consolidation for contextual and auditory fear conditioning is dependent on protein synthesis, PKA, and MAP kinase. Learn Mem 6:97-110.

Siapas AG, Lubenov EV, Wilson MA (2005) Prefrontal phase locking to hippocampal theta oscillations. Neuron 46:141-151.

Squire LR (1992) Memory and the hippocampus: a synthesis from findings with rats, monkeys, and humans. Psychol Rev 99:195-231.

Squire LR (2004) Memory systems of the brain: a brief history and current perspective. Neurobiol Learn Mem 82:171-177.

Squire LR, Alvarez P (1995) Retrograde amnesia and memory consolidation: a neurobiological perspective. Curr Opin Neurobiol 5:169-177.

Wiltgen BJ, Sanders MJ, Anagnostaras SG, Sage JR, Fanselow MS (2006) Context fear learning in the absence of the hippocampus. J Neurosci 26:5484-5491.

Young SL, Bohenek DL, Fanselow MS (1994) NMDA processes mediate anterograde amnesia of contextual fear conditioning induced by hippocampal damage: immunization against amnesia by context preexposure. Behav Neurosci 108:19-29.

Zola-Morgan SM, Squire LR (1990) The primate hippocampal formation: evidence for a time-limited role in memory storage. Science 250:288-290. 